\title{
Regulación del transporte y accidentes de tránsito por vehículos motorizados en el Perú
}

\author{
Regulation of transport and traffic accidents by motor vehicles in Peru \\ Celso Bambarén ${ }^{1}$, Meilind Chú ${ }^{2}$
}

\section{RESUMEN}

Entre 1998 y 2012, en el Perú se han producido más de un millón doscientos mil accidentes terrestres por vehículos automotores con 49 mil fallecidos. A pesar de la aprobación de diversas regulaciones sobre tránsito y seguridad vial, no se observa una tendencia hacia la disminución de las lesiones fatales en los usuarios del tránsito. Las regulaciones parecen no tener un efecto de protección sobre los ocupantes de los vehículos y peatones, debido a que el número de lesiones fatales se ha incrementado en ambos grupos. Las estadísticas podrían tener variaciones del tipo de usuarios afectados por los accidentes y el número de lesiones fatales para los eventos en vías urbanas, peri urbana y carretera.

PALABRAS CLAVE: Accidentes de tránsito, Automóviles, Traumatismo múltiple. (Fuente: DeCS BIREME)

\section{SUMMARY}

Between 1998 and 2012, there have been more than one million two hundred thousand land motor vehicle accidents with 49000 deaths in Peru. Despite the adoption of several regulations on traffic and road safety, there is no a trend towards fewer fatal injuries. Regulations appear not to have a protective effect on the occupants of vehicles and pedestrians, because the number of fatal injuries has increased in both groups. The statistics might have variations on the type of users, affected by the number of accidents, and fatal injuries related to the events in urban and periurban roads, and highways

KEY WORDS: Accidents, Traffic, Automobiles, Multiple trauma. (Source: MeSH NLM)

\section{INTRODUCCIÓN}

Cada año, los accidentes de tránsito causan la muerte de aproximadamente 1,3 millones de personas en todo el mundo y son la causa principal de muerte en el grupo de 15 a 29 años de edad (1). Los países de ingresos medios tienen las mayores tasas de mortalidad, con valores de 20,1 por 100000 habitantes; el Perú en el año 2012 registro 13,73, comparado con 8,7 en los países de ingresos elevados y 18,3 en los de ingresos bajos (2). Entre 1998 al 2012, se han registrado 1 205552 accidentes de tránsito terrestres con 49364 víctimas fatales en el Perú (2). En este mismo periodo, el índice de motorización se incrementó de 0,04 a 0,07 (3).

La prevención de los traumatismos por tránsito requiere que los gobiernos establezcan y hagan cumplir las leyes para garantizar la seguridad de las calles, los vehículos y los usuarios de la vía pública. Unicamente el $15 \%$ de los países cuentan con leyes relacionadas con los cinco factores de riesgo, velocidad excesiva, conducción bajo los efectos del alcohol, uso del casco por los motociclistas, utilización de los cinturones de

\footnotetext{
1 Doctor en Medicina. Profesor Principal, Facultad de Salud Pública y Administración. Universidad Peruana Cayetano Heredia. Lima, Perú.

2 Médico - Cirujano. Centro Médico Naval. Lima, Perú.
} 
seguridad y empleo de medios de sujeción para los niños (4). En el tema del tránsito, también hay que considerar los efectos de las regulaciones, los cuales podrían ser en muchos casos neutros o contrarios a lo esperado, con lo cual se reduce en forma total o parcial los beneficios de las normas. Este fenómeno se conoce como efecto Peltzman, observado en la comparación del número de muertos por accidentes de tránsito, antes y después de la implementación de la utilización de los cinturones de seguridad en los Estados Unidos de Norteamérica (5). Este efecto mostró que la utilización del cinturón de seguridad alentó comportamientos más arriesgados en los conductores y el traslado del mayor riesgo de lesiones a los peatones.

Elobjetivo del estudio fue identificar los dispositivos legales vigentes sobre transporte y seguridad vial relacionados a la prevención de accidentes de tránsito y reducción de las lesiones generadas por estos, y el comportamiento de la accidentabilidad por tránsito terrestre en el país.

\section{CONTENIDO}

Se revisaron las normas de tránsito emitidas por el gobierno peruano en los últimos 15 años vinculados al transporte y la seguridad vial publicadas en el Diario Oficial El Peruano, siendo seleccionados aquellos dispositivos de aplicación nacional y vinculados a los cinco factores de riesgo para prevenir lesiones por accidentes. Se encontró que el gobierno aprobó diversas normas con la finalidad de regular el tránsito terrestre en el país. En 1999, apareció la Ley 27181 Ley General de transporte y tránsito terrestre, que estableció las competencias de las autoridades, el registro vehicular y la obligatoriedad del seguro de accidentes de tránsito (6), pero recién se implementó en el 2002 (7). El reglamento nacional de tránsito aprobado en 2001 incluyó el uso obligatorio del cinturón de seguridad para los ocupantes de los asientos delanteros (piloto y copiloto), restricciones para los usuarios y las características de la señalización en las vías (8). Esta norma fue modificada en 2006 añadiendo el uso obligatorio del cinturón de seguridad en los asientos posteriores de los vehículos particulares (9) y en los asientos posteriores de los vehículos del servicio de transporte Interprovincial de personas (10).

En 2009 se aprobó el Texto único del reglamento de tránsito - código de tránsito (11), siendo modificado el mismo año para incorporar regulaciones sobre número máximo de pasajeros en vehículos, utilización de las vías por los vehículos menores, paraderos, uso de cinturones de seguridad y cabezales en vehículos de transporte interprovincial y de carga, incorporación de los cinturones de seguridad en los vehículos menores de 3 ruedas, incremento de sanciones a conductores y sanciones preventivas para peatones (12). Posteriormente, una nueva modificación al código de tránsito en el año 2010, incorporó las multas y sanciones pecuniarias a los peatones (13). Otra norma importante es el reglamento nacional de vehículos que establece los requisitos técnicos de los vehículos que para circular por las vías terrestres y la obligatoriedad de las revisiones técnicas vehiculares (14) y el Reglamento Nacional de licencias de conducir de vehículos automotores y no motorizados aprobada en el 2008 (15) y modificada en 2010 (13). También, se emitió una norma específica para prevenir los accidentes en las carreteras causados por los vehículos interprovinciales y de mercancías mediante el control de las requisitos legales y condiciones mecánicas de los medios de transporte en las garitas de peaje denominado "tolerancia cero" (16).

Al año 2013, las normas aprobadas e implementadas abarcan cuatro de los cinco factores de riesgo, no habiendo sido todavía regulado el empleo de medios de sujeción para los niños en los vehículos. A pesar de ello, según las estadísticas de accidentes de la Policía Nacional muestra que entre 1998 y 2004, disminuyo el número de los accidentes pero sin reducción de los fallecidos, luego de lo cual el número de accidentes ha continuado aumentando mientras que la mortalidad por este tipo de eventos ha tenido un comportamiento irregular (2).

\section{DISCUSIÓN}

En el caso peruano, uno de los factores de mayor relevancia para sustentar el incremento de los accidentes es el crecimiento desordenado, inseguro e indiscriminado del parque automotor (17). El índice de motorización aumentó de 41,92 por 1000 habitantes en 1998 a 70,94 en 2012 (Gráfico 1). Los accidentes y las lesiones fatales muestran una marcada disminución entre 1998 y 2003, luego continúan disminuyendo pero en forma más lenta hasta el 2011 donde la mortalidad nuevamente comienza a incrementarse. El número de muertos por vehículos en el Perú está por encima de países como México, Colombia, Chile y Argentina que tienen un parque automotor más grande, Chile con 7 por 10000 vehículos o Argentina con valores de 5. Como explicar que a pesar de las numerosas regulaciones aprobadas, la accidentabilidad y sus efectos no hayan variado en los últimos años. Una 
Gráfico 1. Número de accidentes de tránsito y lesiones fatales por 10000 vehículos Perú. 1998 a 2012.

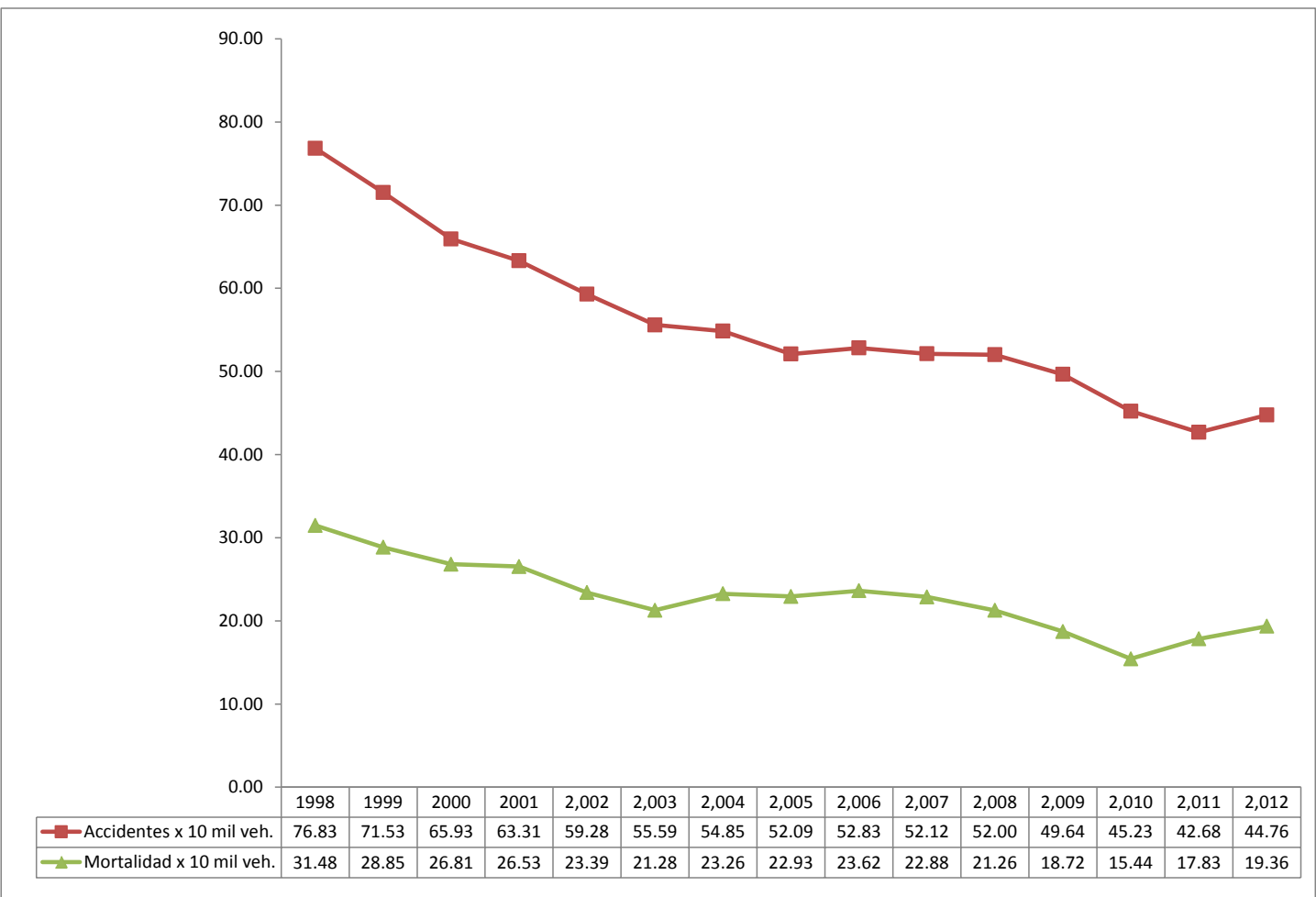

Gráfico 2. Accidentes de tránsito generados por el conductor, peatón y por otras causas en el Perú. 1998 a 2012.

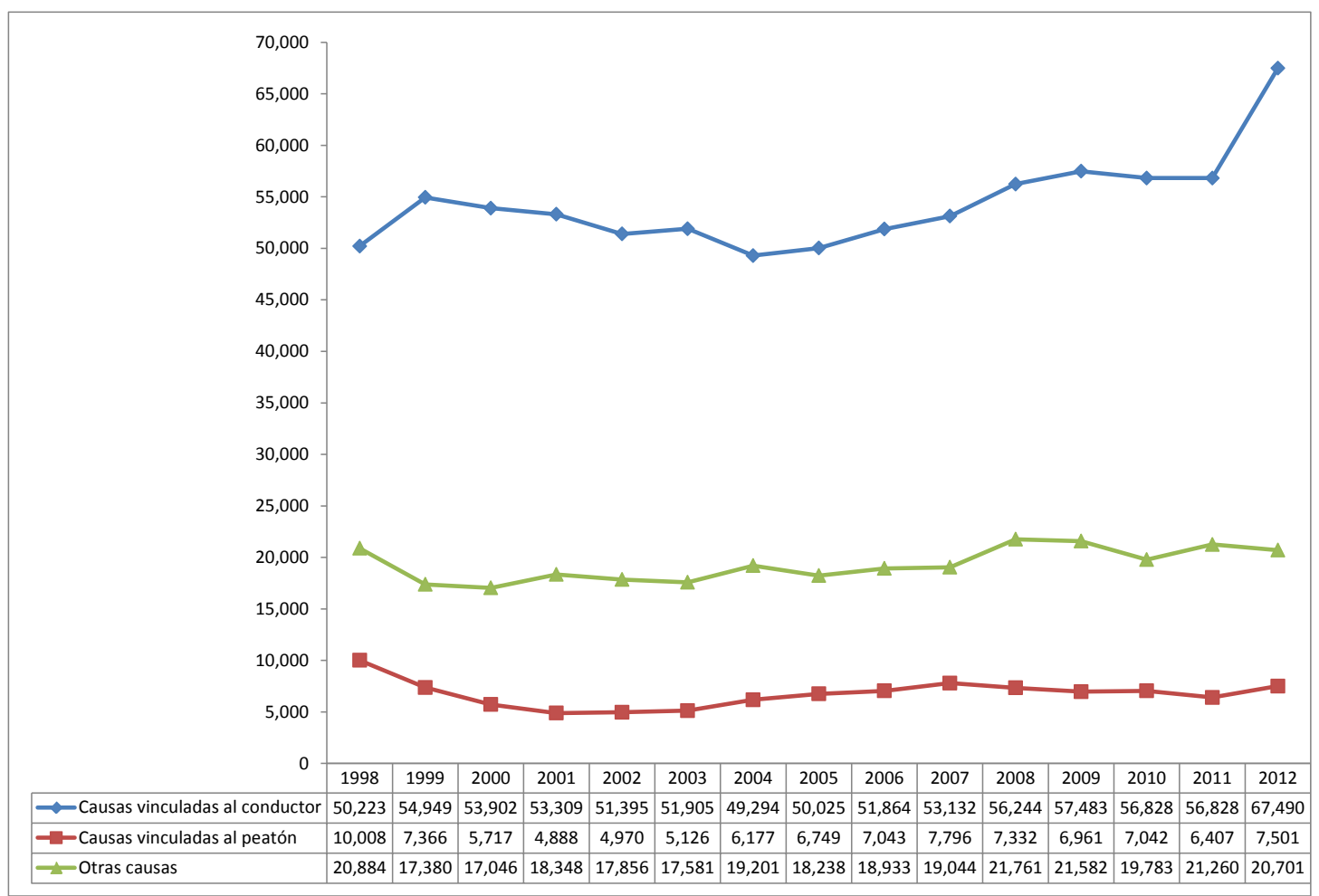


primera aproximación a una explicación es considerar el efecto Peltzman, es decir la reacción frente a una regulación de seguridad aumentando otros comportamientos de riesgo, lo que neutraliza total o parcialmente los beneficios de las regulaciones (14) (Gráfico 2).

En el caso peruano, no se observa una tendencia hacia la disminución de los accidentes y de las muertes que estos producen, a pesar de la implementación de numerosas normas en los últimos diez años. Los accidentes causados por la imprudencia, exceso de velocidad y ebriedad del conductor se redujeron entre 1998 y 2004, luego de lo cual han mantenido un incremento en forma sostenida, similar situación sucedió con los eventos causados por la imprudencia de los peatones. Ello, podría vincularse con la implementación, en la primeros cinco años del siglo XXI, de la ley de transporte y tránsito, y los reglamentos nacionales de tránsito y vehículos, luego de lo cual no se ha vuelto a repetir esta tendencia hacia la disminución de los accidentes y las lesiones fatales, a pesar de la aprobación de sanciones más severas para los usuarios del tránsito (Gráfico 3).

Entre 2001 y 2005, se produjo una disminución de los choques y volcaduras que afectan a los ocupantes de los vehículos automotores, mientras que los atropellos se incrementaron. Los efectos positivos en la reducción de accidentes en los ocupantes de los vehículos fueron neutralizados por el incremento de los eventos en los peatones. No se dispone de información sobre lesiones fatales en ocupantes de vehículos y peatones, con lo cual se hubiese podido refutar o no la observación de Peltzman sobre que las regulaciones fomentan que los conductores tomen mayores riesgos, debido a contar con exigencias de protección como los cinturones de seguridad, lo que se evidencia en un incremento de accidentes con la disminución de las lesiones fatales, mientras se incrementan los accidentes y muertes de los peatones. Las estadísticas de los accidentes y lesiones fatales en la red vial nacional registradas por la policía de carreteras, cuatro años antes y después de la implementación de la "tolerancia cero" no mostraron un efecto positivo de la regulación. En los cuatros años previos a la implementación de esta norma, los accidentes disminuyeron $25 \%$ en promedio, mientras que en los cuatro años posteriores se incrementaron en 3\%. Las lesiones fatales luego de la aplicación de la norma, se incrementaron $2 \%$ en promedio, mientras que en los cuatro años previos a la regulación, los fallecidos disminuyeron en $8 \%$.

Los resultados del estudio tuvieron como limitaciones que no se dispone de información para evaluar el grado del control del cumplimiento de las regulaciones por parte de la Policía Nacional y las autoridades; así como no se dispone de datos desagregados sobre lesiones en los diferentes usuarios de tránsito y según tipo de vías.

Gráfico 3. Número de accidentes de tránsito en peatones y ocupantes de vehículos automotores. Perú. 1998 a 2012.

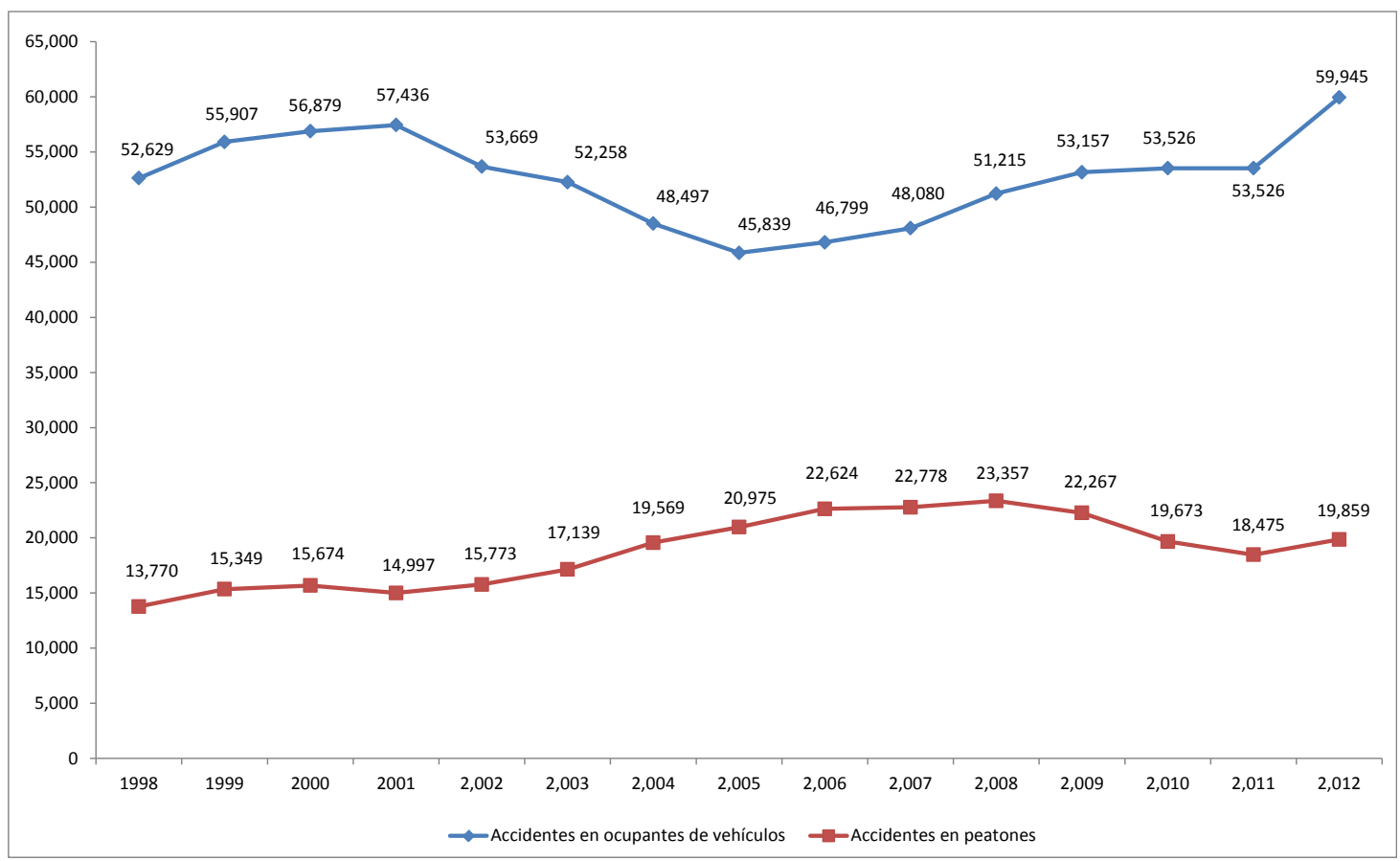


Con base en la revisión realizada y las estadísticas de las lesiones de tránsito, se concluye que la cantidad absoluta de los accidentes y las lesiones fatales no registran una tendencia a la disminución sino que a pesar de las regulaciones y de la severidad de las sanciones para quienes incumplen las normas de tránsito, la cantidad de fallecidos tienden a incrementarse especialmente en los últimos dos años. En algunos casos, es esperable que los accidentes de tránsito pudiesen incrementarse acompañado de una disminución de las lesiones fatales. Ello, podría estar ocurriendo en los sucesos de tránsito en las zonas urbanas donde diversos dispositivos y elementos arquitectónicos protegen más al peatón y limitan la posibilidad de exceso de velocidad e imprudencia del conductor. Mientras que en las zonas peri urbanas, vías de alta velocidad y en las zonas rurales podrían estar contribuyendo con un mayor número de usuarios de tránsito fallecidos. En tal sentido, es importante que para el diseño de las políticas públicas de seguridad vial es necesario considerar las recomendaciones de los estudios de Peltzman sobre la necesidad previa de evaluar el impacto efectivo que tendría las regulaciones en el comportamiento de los usuarios del tránsito, y luego cuando las norma estén implementadas, verificar si la regulación cumplió con los fines para los cuales estuvo dirigida o si genero efectos negativos (5).

\section{Declaración de financiamiento y de conflictos de intereses:}

Los autores declaran que no han recibido algún tipo de financiamiento para la realización de la presente investigación, y que no tienen conflictos de orden económico, institucional, laboral o personal.

\section{Correspondencia:}

Celso Bambarén

Loma Verde 130 - Lima 33 Perú

Correo electrónico: celso.bambaren.a@upch.pe

\section{REFERENCIAS BIBLIOGRÁFICAS}

1. World Health Organization. Global status report on road safety 2013. Supporting a decade of action. Luxembourg: WHO; 2013.

2. Consejo Nacional de Seguridad Vial. Cuadros estadísticos. (Citado el 5 de agosto de 2013). Disponible en: URL: http://www.mtc.gob.pe/cnsv/ estadisticas/index.html

3. Ministerio de Transportes y Comunicaciones. Parque vehicular nacional estimado por años. (Citado el 2 de agosto de 2013). Disponible en URL: http://www. mtc.gob.pe/estadisticas/t-carretero.html

4. Organización Mundial de la Salud. Traumatismos causados por el tránsito. Centro de prensa. Nota descriptiva $\mathrm{N}^{\circ}$ 358. (Citado el 24 de octubre de 2013). Disponible en URL: http://www.who.int/ mediacentre/factsheets/fs358/es/

5. Peltzman, S. Regulation and the Wealth of Nations: The Connection between Government Regulation and Economic Progress. (Citado octubre 24, 2013). Disponible en URL: http://pcpe.libinst.cz/nppe/3_2/ nppe3_2_3.pdf

6. Congreso de la Republica. Ley General de transporte y tránsito terrestre. Ley 27181. Diario Oficial El Peruano. Lima, Perú. 1999, 07 de octubre.

7. Ministerio de Transporte y Comunicaciones. Texto Único Ordenado del Reglamento Nacional de Responsabilidad Civil y Seguros Obligatorios por Accidentes de Tránsito. Decreto Supremo 024-2002MTC. Diario Oficial El Peruano. Lima, Perú. 2002, 14 de junio.

8. Ministerio de Transporte y Comunicaciones. Reglamento Nacional de Tránsito. Decreto Supremo 033-2001-MTC. Diario oficial El Peruano. Lima, Perú. 2001, 10 de agosto.

9. Ministerio de Transporte y Comunicaciones. Modificación del Reglamento Nacional de Tránsito. Decreto Supremo 027-2006-MTC. Diario oficial El Peruano. Lima, Perú. 2006, 22 de julio.

10. Ministerio de Transporte y Comunicaciones. Modificación del Reglamento Nacional de Tránsito. Decreto Supremo 032-2006-MTC. Diario oficial El Peruano. Lima, Perú. 2006, 30 de setiembre.

11. Ministerio de Transporte y Comunicaciones. Texto único del reglamento de tránsito, Código de tránsito. Decreto Supremo 016-2009-MTC. Diario oficial El Peruano. Lima, Perú. 2009, 22 de abril.

12. Ministerio de Transporte y Comunicaciones. Modifican el texto único del reglamento de tránsito - Código de tránsito. Decreto Supremo 029-2009MTC. Diario oficial El Peruano. Lima, Perú. 2009, 20 de julio.

13. Ministerio de Transporte y Comunicaciones. Modificación del texto único del Reglamento de Tránsito aprobado por Decreto Supremo 016-2009MTC, el Decreto Supremo 029-2009-MTC y el Reglamento Nacional de Licencias de Conducir Vehículos Automotores y no Motorizados de Transporte Terrestre, aprobado por Decreto Supremo 040-2008-MTC. Decreto Supremo 040-2010-MTC. Diario oficial El Peruano. Lima, Perú. 2010, 16 de agosto.

14. Ministerio de Transporte y Comunicaciones. Reglamento Nacional de Vehículos. Decreto Supremo 058-2003-MTC. Diario oficial El Peruano. Lima, Perú. 2003, 9 de octubre. 
15. Ministerio de Transporte y Comunicaciones. Reglamento Nacional de Licencias de conducir de vehículos automotores y no motorizados. Decreto Supremo 040-2008-MTC. Diario oficial El Peruano. Lima, Perú. 2008, 18 de noviembre.

16. Ministerio de Transporte y Comunicaciones. Sistema de control en garitas de peaje "tolerancia cero". Decreto Supremo 035-2006-MTC. Diario oficial El Peruano. Lima, Perú. 30 de octubre de 2006.

17. Chia L, Humani S. Accidentes de tránsito en el Perú:
¿Casualidad o causalidad'. Lima, Perú: Ministerio de Transportes y Comunicaciones. Cuadernos de infraestructura e inclusión social. Año 1. No 3. 2010.

18. Peltzam S. Regulation and the natural progress of opulence. New York, USA: The AEI Press; 2005.

Recibido: 02/09/2013

Aceptado: 07/11/2013 